\title{
UPPER BOUNDS FOR THE REGULARITY OF POWERS OF EDGE IDEALS OF GRAPHS
}

\author{
A. V. JAYANTHAN AND S. SELVARAJA
}

\begin{abstract}
Let $G$ be a finite simple graph and $I(G)$ denote the corresponding edge ideal. In this paper, we obtain upper bounds for the Castelnuovo-Mumford regularity of $I(G)^{q}$ in terms of certain combinatorial invariants associated with $G$. We also prove a weaker version of a conjecture by Alilooee, Banerjee, Beyarslan and Hà on an upper bound for the regularity of $I(G)^{q}$ and we prove the conjectured upper bound for the class of vertex decomposable graphs. Using these results, we explicitly compute the regularity of $I(G)^{q}$ for several classes of graphs.
\end{abstract}

\section{INTRODUCTION}

Let $I$ be a homogeneous ideal of a polynomial ring $R=\mathbb{K}\left[x_{1}, \ldots, x_{n}\right]$ over a field $\mathbb{K}$ with usual grading. In [7], Bertram, Ein and Lazarsfeld have initiated the study of the Castelnuovo-Mumford regularity, henceforth denoted as $\operatorname{reg}(-)$, of $I^{q}$ as a function of $q$ by proving that if $I$ is the defining ideal of a smooth complex projective variety, then $\operatorname{reg}\left(I^{q}\right)$ is bounded by a linear function of $q$. Then, Chandler [14] and Geramita, Gimigliano and Pitteloud [19] proved that if $\operatorname{dim}(R / I) \leq 1$, then $\operatorname{reg}\left(I^{q}\right) \leq q \operatorname{reg}(I)$ for all $q \geq 1$. However, Swanson [39] proved that there exists $k \geq 1$ such that for all $q \geq 1, \operatorname{reg}\left(I^{q}\right) \leq k q$. Thereafter, Cutkosky, Herzog and Trung, [16], and independently Kodiyalam [33], proved that for a homogeneous ideal $I$ in a polynomial ring, $\operatorname{reg}\left(I^{q}\right)$ is a linear function for $q \gg 0$ i.e., there exist non negative integers $a$ and $b$ depending on $I$ such that $\operatorname{reg}\left(I^{q}\right)=$ $a q+b$ for all $q \gg 0$. While the coefficient $a$ is well-understood ([16], [33], [40]), the free constant $b$ and the stabilization index $q_{0}=\min \left\{q^{\prime} \mid \operatorname{reg}\left(I^{q}\right)=a q+b\right.$, for all $\left.q \geq q^{\prime}\right\}$ are quite mysterious. Therefore, the attention has been to identify classes for which the linear polynomial can be computed or bounded using invariants associated to $I$. There have been some attempts on computing the free constant and stabilization index for several class of ideals. For instance, if $I$ is a equigenerated homogeneous ideal, then $b$ is related to the regularity of fibers of certain projection map (see for example, [37]). If $I$ is $\left(x_{1}, \ldots, x_{n}\right)$-primary, then $q_{0}$ can be related to partial regularity of the Rees algebra of $I$ (see for example, [6]). In this paper, we study the regularity of powers of edge ideals associated to finite simple graphs.

Let $G$ be a finite simple graph without isolated vertices on the vertex set $\left\{x_{1}, \ldots, x_{n}\right\}$ and $I(G):=\left(\left\{x_{i} x_{j} \mid\left\{x_{i}, x_{j}\right\} \in E(G)\right\}\right) \subset \mathbb{K}\left[x_{1}, \ldots, x_{n}\right]$ be the edge ideal corresponding to the graph $G$. It is known that $\operatorname{reg}\left(I(G)^{q}\right)=2 q+b$ for some $b$ and $q \geq q_{0}$. There are very few classes of graphs for which $b$ and $q_{0}$ are known. We refer the reader to [3]

Key words and phrases. Castelnuovo-Mumford regularity, powers of edge ideals, vertex decomposable graphs.

AMS Classification 2010: 13D02, 13F20, 05C25. 
and the references cited there for a review of results in the literature in this direction. While the aim is to obtain the linear polynomial corresponding to $\operatorname{reg}\left(I(G)^{q}\right)$, it seems unlikely that a single combinatorial invariant will represent the constant term for all graphs. This naturally give rise to two directions of research. One direction is to obtain linear polynomials for particular classes of graphs. Another direction is to obtain upper and lower bounds for $\operatorname{reg}\left(I(G)^{q}\right)$ using combinatorial invariants associated to the graph $G$. It was proved by Beyarslan, Hà and Trung that $2 q+\nu(G)-1 \leq \operatorname{reg}\left(I(G)^{q}\right)$ for all $q \geq 1$, where $\nu(G)$ denotes the induced matching number of $G$, [8]. In [29], the authors along with Narayanan proved that for a bipartite graph $G, \operatorname{reg}\left(I(G)^{q}\right) \leq 2 q+\operatorname{co-chord}(G)-1$ for all $q \geq 1$, where co-chord $(G)$ denote the co-chordal cover number of $G$. There is no general upper bound known for powers of edge ideals of arbitrary graphs. Therefore, one may ask:

Q1. Does there exists a function $\rho:\{$ finite simple graphs $\} \rightarrow \mathbb{N}$ such that for any given graph $G, \operatorname{reg}\left(I(G)^{q}\right) \leq 2 q+\rho(G)$ for all $q \geq 1$.

Q2. Can one obtain the linear polynomial corresponding to $\operatorname{reg}\left(I(G)^{q}\right)$ for various classes of graphs?

This paper evolves around these two questions.

The first main result of the paper answers Question Q1. We prove that if the numerical function $\rho$ satisfies certain properties, then an upper bound as in Q1 is true:

We first fix a notation that we consider throughout this paper. Let $G$ be a graph and $\mathcal{I}_{G}$ be the set of all non-empty induced subgraphs of $G$.

Theorem 4.1. Let $G$ be a graph and $\rho: \mathcal{I}_{G} \longrightarrow \mathbb{N}$ be a function such that for any $L \in \mathcal{I}_{G}$,

(1) $\operatorname{reg}(I(L)) \leq \rho(L)+1$,

(2) $\rho\left(L_{1}\right) \leq \rho(L)$ for any induced subgraph $L_{1}$ of $L$ and

(3) there exists a vertex $x \in V(L)$ such that $\rho\left(L \backslash N_{L}[x]\right)+1 \leq \rho(L)$.

Then

$$
\operatorname{reg}\left(I(G)^{q}\right) \leq 2 q+\rho(G)-1 \text { for all } q \geq 1
$$

As an application of this result, we obtain upper bounds in terms of certain specific combinatorial invariants. Hà and Woodroofe [23] defined an invariant in terms of star packing, denoted by $\zeta(G)$ (see Section 4 for the definition), and proved that $\operatorname{reg}(I(G)) \leq$ $\zeta(G)+1$. Also, Woodroofe proved that $\operatorname{reg}(I(G)) \leq \operatorname{co-chord}(G)+1$. Alilooee, Banerjee, Beyarslan and Há conjectured ([3, Conjecture 7.11(1)]):

Conjecture 1.1. Let $G$ be a graph. Then for all $q \geq 1, \operatorname{reg}\left(I(G)^{q}\right) \leq 2 q+\operatorname{co-chord}(G)-1$.

In this paper, we prove Conjecture 1.1. We also extend the result by Hà and Woodroofe to all powers.

Theorem 4.4. Let $G$ be a graph. Then for all $q \geq 1$,

(1) $\operatorname{reg}\left(I(G)^{q}\right) \leq 2 q+\zeta(G)-1$.

(2) $\operatorname{reg}\left(I(G)^{q}\right) \leq 2 q+\operatorname{co}-\operatorname{chord}(G)-1$. 
Another way of bounding the function $\operatorname{reg}\left(I(G)^{q}\right)$, than using combinatorial invariants, is to relate it to the regularity of $G$ itself. It was conjectured by Alilooee, Banerjee, Beyarslan and Hà, [3, Conjecture 7.11(2)]:

Conjecture 1.2. If $G$ is a graph, then for all $q \geq 1, \operatorname{reg}\left(I(G)^{q}\right) \leq 2 q+\operatorname{reg}(I(G))-2$.

There are some classes of graphs for which this conjecture is known to be true, see [3, 5]. As a consequence of the techniques that we have developed, we prove the conjecture with an additional hypothesis:

Corollary 4.2. Let $G$ be a graph. If every induced subgraph $H$ of $G$ has a vertex $x$ with $\operatorname{reg}\left(I\left(H \backslash N_{H}[x]\right)\right)+1 \leq \operatorname{reg}(I(H))$, then for all $q \geq 1$,

$$
\operatorname{reg}\left(I(G)^{q}\right) \leq 2 q+\operatorname{reg}(I(G))-2 .
$$

We recover many of the known results on the regularity of powers of edge ideals of graphs (Corollary 4.5). Also, as a consequence of our results we answer Q2 by obtaining precise expressions for the regularity of powers of edge ideals of some classes of graphs, (Proposition 4.7, Proposition 4.8).

So far, in the literature, for the classes of graphs for which the regularity of powers of edge ideals have been computed, they satisfy either $\operatorname{reg}\left(I(G)^{q}\right)=2 q+\nu(G)-1$ or $\operatorname{reg}\left(I(G)^{q}\right)=2 q+\operatorname{co-chord}(G)-1$, for all $q \geq 2$. In [29], the authors raised the question whether there exists a graph $G$ with

$$
2 q+\nu(G)-1<\operatorname{reg}\left(I(G)^{q}\right)<2 q+\operatorname{co}-\operatorname{chord}(G)-1, \text { for } q \gg 0 .
$$

As a consequence of our investigation, we obtain a class of graphs for which

$$
2 q+\nu(G)-1<\operatorname{reg}\left(I(G)^{q}\right)=2 q+\zeta(G)-1<2 q+\operatorname{co-chord}(G)-1, \text { for } q \gg 0 .
$$

We then proceed to prove the Conjecture 1.2 for vertex decomposable graphs. A graph $G$ is said to be vertex decomposable if $\Delta(G)$ is vertex decomposable, where $\Delta(G)$ denotes the independence complex of $G$ (see Section 5 for the definition). Vertex decomposability of simplicial complexes was first introduced by Provan and Billera [36], in the case when all the maximal faces are of the same cardinality, and extended to the arbitrary case by Björner and Wachs [11. We have the chain of implications

vertex decomposable $\Longrightarrow$ shellable $\Longrightarrow$ sequentially Cohen-Macaulay.

A graph $G$ is said to be shellable if $\Delta(G)$ is a shellable simplicial complex and $G$ is sequentially Cohen-Macaulay if $R / I(G)$ is sequentially Cohen-Macaulay. Both the above implications are known to be strict. Recently, a number of authors have been interested in classifying or identifying vertex decomposable graphs $G$ in terms of the combinatorial properties of $G$, see $[9,10,32,42,43,44]$.

Theorem 5.3. If $G$ is a vertex decomposable graph, then for all $q \geq 1$,

$$
\operatorname{reg}\left(I(G)^{q}\right) \leq 2 q+\operatorname{reg}(I(G))-2 .
$$

Banerjee, Beyarslan and Hà gave a question whether the equality $\operatorname{reg}\left(I(G)^{q}\right)=2 q+$ $\nu(G)-1$ hold for all $q \geq 1$ for several important classes of graphs, [3, Question 7.9]. We answer this question affirmatively in Corollary 5.4. 
Our paper is organized as follows. In Section 2, we collect the terminology and preliminary results that are essential for the rest of the paper. We prove, in Section 3, several technical lemmas which are needed for the proof of our main results which appear in Sections 4 and 5 .

\section{Notation AND PRELIMINARIES}

Throughout this article, $G$ denotes a finite simple graph without isolated vertices. For a graph $G$, let $V(G)$ and $E(G)$ denote the set of all vertices and the set of all edges of $G$, respectively. The degree of a vertex $x \in V(G)$, denoted by $\operatorname{deg}_{G}(x)$, is the number of edges incident to $x$. A subgraph $H \subseteq G$ is called induced if for $u, v \in V(H),\{u, v\} \in E(H)$ if and only if $\{u, v\} \in E(G)$. For $\left\{u_{1}, \ldots, u_{r}\right\} \subseteq V(G)$, let $N_{G}\left(u_{1}, \ldots, u_{r}\right)=\{v \in$ $V(G) \mid\left\{u_{i}, v\right\} \in E(G)$ for some $\left.1 \leq i \leq r\right\}$ be the set of neighbors of $u_{1}, \ldots, u_{r}$ and $N_{G}\left[u_{1}, \ldots, u_{r}\right]=N_{G}\left(u_{1}, \ldots, u_{r}\right) \cup\left\{u_{1}, \ldots, u_{r}\right\}$. For $U \subseteq V(G)$, we denote by $G \backslash U$ the induced subgraph of $G$ on the vertex set $V(G) \backslash U$. Let $C_{n}$ denote the cycle on $n$ vertices.

A subset $X$ of $V(G)$ is called independent if there is no edge $\{x, y\} \in E(G)$ for $x, y \in X$. A matching in a graph $G$ is a subgraph consisting of pairwise disjoint edges. If a collection of pairwise disjoint edges is an induced subgraph, then the matching is said to be an induced matching. The largest size of an induced matching in $G$ is called its induced matching number and denoted by $\nu(G)$.

One important tool in the study of regularity of powers of edge ideals is even-connections. We recall the concept of even-connectedness from [2].

Definition 2.1. Let $G$ be a graph. Two vertices $u$ and $v$ ( $u$ may be the same as $v$ ) are said to be even-connected with respect to an $s$-fold products $e_{1} \cdots e_{s}$, where $e_{1}, \ldots, e_{s}$ are edges of $G$, not necessarily distinct, if there is a path $p_{0} p_{1} \cdots p_{2 k+1}, k \geq 1$ in $G$ such that:

(1) $p_{0}=u, p_{2 k+1}=v$.

(2) For all $0 \leq l \leq k-1, p_{2 l+1} p_{2 l+2}=e_{i}$ for some $i$.

(3) For all $i,\left|\left\{l \geq 0 \mid p_{2 l+1} p_{2 l+2}=e_{i}\right\}\right| \leq\left|\left\{j \mid e_{j}=e_{i}\right\}\right|$.

(4) For all $0 \leq r \leq 2 k, p_{r} p_{r+1}$ is an edge in $G$.

Remark 2.2. While we understand that the definition of even-connection requires $k \geq 1$, for convenience of writing the proofs, we consider an edge to be trivially even-connected, i.e., we take the even-connection by setting $k=0$ in the above definition.

The following theorem due to Banerjee is used repeatedly throughout this paper:

Theorem 2.3. [2, Theorem 6.1 and Theorem 6.7] Let $G$ be a graph with edge ideal $I=I(G)$, and let $s \geq 1$ be an integer. Let $M$ be a minimal generator of $I^{s}$. Then $\left(I^{s+1}: M\right)$ is minimally generated by monomials of degree 2, and uv ( $u$ and $v$ may be the same) is a minimal generator of $\left(I^{s+1}: M\right)$ if and only if either $\{u, v\} \in E(G)$ or $u$ and $v$ are even-connected with respect to $M$.

Polarization is a process to obtain a squarefree monomial ideal from a given monomial ideal.

Definition 2.4. Let $M=x_{1}^{a_{1}} \cdots x_{n}^{a_{n}}$ be a monomial in $R=\mathbb{K}\left[x_{1}, \ldots, x_{n}\right]$. Then we define the squarefree monomial $P(M)$ (polarization of $M$ ) as

$$
P(M)=x_{11} \cdots x_{1 a_{1}} x_{21} \cdots x_{2 a_{2}} \cdots x_{n 1} \cdots x_{n a_{n}}
$$


in the polynomial ring $R_{1}=\mathbb{K}\left[x_{i j} \mid 1 \leq i \leq n, 1 \leq j \leq a_{i}\right]$. If $I=\left(M_{1}, \ldots, M_{q}\right)$ is an ideal in $R$, then the polarization of $I$, denoted by $\widetilde{I}$, is defined as $\widetilde{I}=\left(P\left(M_{1}\right), \ldots, P\left(M_{q}\right)\right)$.

Let $G$ be a graph on the vertex set $V(G)=\left\{x_{1}, \ldots, x_{n}\right\}$ and $I(G) \subset R=\mathbb{K}\left[x_{1}, \ldots, x_{n}\right]$ denote the edge ideal of $G$. For an edge $e=\left\{x_{i}, x_{j}\right\}$, we consider $e=x_{i} x_{j}$ as an element of the polynomial ring $R$. Let $M$ be a minimal monomial generator of $I(G)^{s}$. Then $M$ can be written as product of $s$ edges, i.e., $M=e_{1} \cdots e_{s}$, for some edges $e_{1}, \ldots, e_{s}$, not necessarily distinct. By Theorem 2.3, $J=\left(I(G)^{s+1}: e_{1} \cdots e_{s}\right)$ is a quadratic monomial ideal. If $x_{i} x_{j}$ is a minimal generator of $J$, then $x_{i}$ and $x_{j}$ correspond to the vertices of $G$ which are even-connected with respect to $e_{1} \cdots e_{s}$. If $x_{i}=x_{j}$, then $x_{i}^{2}$ is a minimal generator of $J$. We consider $\tilde{J}$, the polarization of $J$, contained in the ring $R_{1}=\mathbb{K}\left[x_{1}, \ldots, x_{n}, z_{1}, \ldots, z_{n}\right]$ such that if $x_{i}^{2} \in J$, then $P\left(x_{i}^{2}\right)=x_{i} z_{i} \in \tilde{J}$. By considering $V^{\prime}=\left\{x_{1}, \ldots, x_{n}, z_{1}, \ldots, z_{n}\right\}$ as vertices, one can see that $\tilde{J}$ corresponds to a graph, $G^{\prime}$, on a vertex set $V\left(G^{\prime}\right) \subseteq V^{\prime}$. First note that $I(G) \subset J \subset \tilde{J}$, by considering all these ideals in $R_{1}$. Consequently, we can consider $G$ as a subgraph of $G^{\prime}$. Note that $G$ may not be an induced subgraph of $G^{\prime}$.

For example, let $G=C_{5}$ and $I(G)=\left(x_{1} x_{2}, x_{2} x_{3}, x_{3} x_{4}, x_{4} x_{5}, x_{5} x_{1}\right) \subset \mathbb{K}\left[x_{1}, \ldots, x_{5}\right]$. Let $M=x_{2} x_{3} x_{4} x_{5}$ be a minimal monomial generator of $I(G)^{2}$. Then $\left(I(G)^{3}: M\right)=$

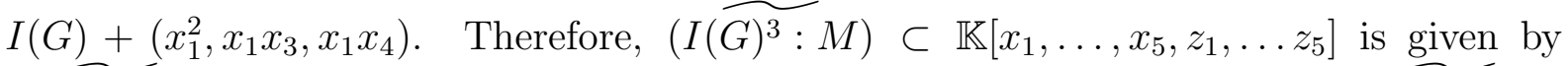
$\left(I\left(\widetilde{\left.G)^{3}: M\right)}\right) I(G)+\left(x_{1} z_{1}, x_{1} x_{3}, x_{1} x_{4}\right)\right.$. Let $G^{\prime}$ be the graph associated to $\left(I\left(\widetilde{\left.G)^{3}: M\right)}\right)\right.$. Then $V\left(G^{\prime}\right)=V(G) \cup\left\{z_{1}\right\}$ and $E\left(G^{\prime}\right)=E(G) \cup\left\{\left\{x_{1}, z_{1}\right\},\left\{x_{1}, x_{3}\right\},\left\{x_{1}, x_{4}\right\}\right\}$. Note also that $N_{G}\left(x_{1}\right)=\left\{x_{2}, x_{5}\right\}$ and $N_{G^{\prime}}\left(x_{1}\right)=\left\{x_{2}, x_{3}, x_{4}, x_{5}, z_{1}\right\}$.

For details of polarization we refer the reader to [24]. In this paper, we repeatedly use one of its important properties, namely:

Corollary 2.5. [24, Corollary 1.6.3(a)] Let I be a monomial ideal in $\mathbb{K}\left[x_{1}, \ldots, x_{n}\right]$. Then $\operatorname{reg}(I)=\operatorname{reg}(\widetilde{I})$.

\section{TECHNICAL LEMMAS}

In this section, we prove several technical results concerning the graph associated with $\left(I(G)^{s+1}: e_{1} \cdots e_{s}\right)$ and some of its induced subgraphs. We begin by fixing the notation for the most of our results.

Notation 3.1. Let $G$ be a graph with $V(G)=\left\{x_{1}, \ldots, x_{n}\right\}$ and $e_{1}, \ldots, e_{s}, s \geq 1$, be some

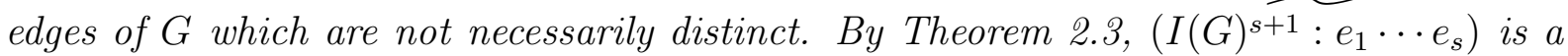
quadratic squarefree monomial ideal in an appropriate polynomial ring. We denote by $G^{\prime}$ the graph associated to $\left(I(G)^{s+1: e_{1}} \cdots e_{s}\right)$.

One of the key ingredients in the proof of the main results is a new graph, $G^{\prime}$, obtained from a given graph $G$ as in Notation 3.1. Our main aim in this section is to get an upper bound for regularity of certain induced subgraphs of $G^{\prime}$ which in turn will help us in bounding $\operatorname{reg}\left(I\left(G^{\prime}\right)\right)$. For this purpose, we need to understand the structure of the graph $G^{\prime}$ in more detail. First we show that whiskers can be ignored when taking even-connections. 
Lemma 3.2. Let $G$ be a graph and $e_{1}, \ldots, e_{s} \in E(G)$ where $s \geq 1$. Assume that for some $1 \leq i \leq s, e_{i}=\{x, y\}$ with $N_{G}(x)=\{y\}$. Then

$$
\left(I(G)^{s+1}: e_{1} \cdots e_{s}\right)=\left(I(G)^{s}: \prod_{j \neq i} e_{j}\right)
$$

Proof. By [34, Lemma 2.10], we have $\left(I(G)^{s+1}: x y\right)=I(G)^{s}$ for all $s \geq 1$. Hence $\left(I(G)^{s+1}: e_{1} \cdots e_{s}\right)=\left(\left(I(G)^{s+1}: e_{i}\right): \prod_{j \neq i} e_{j}\right)=\left(I(G)^{s}: \prod_{j \neq i} e_{j}\right)$.

The following result shows that if a vertex has no intersection with a set of edges, then removing such a vertex and taking even-connection with respect to the set of those edges commute with each other.

Lemma 3.3. We use the notation in Notation 3.1. Let $G$ be a graph and $e_{1}, \ldots, e_{s} \in E(G)$ where $s \geq 1$. If $x \in V(G)$ satisfy $\{x\} \cap e_{i}=\emptyset$ for all $1 \leq i \leq s$, then

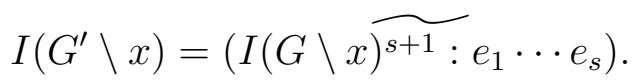

Proof. Clearly $\left(I\left(G \backslash x \widetilde{)^{s+1}:} e_{1} \cdots e_{s}\right) \subseteq I\left(G^{\prime} \backslash x\right)\right.$. Let $u, v \in V(G \backslash x)$, not necessarily distinct, be such that $u$ is even-connected to $v$ in $G$ with respect to $e_{1} \cdots e_{s}$. Let $(u=$ $\left.p_{0}\right) p_{1} \cdots p_{2 k}\left(p_{2 k+1}=v\right)$ be an even-connection in $G$. Since $e_{i} \cap\{x\}=\emptyset$ for all $1 \leq i \leq s$, $u$ is even-connected to $v$ in $G \backslash x$ with respect to $e_{1} \cdots e_{s}$.

The next two results which throws more light into the structure of $G^{\prime}$ have been proved in [30]. While in [30], the hypothesis was that the graph is very well-covered, it may be noted that these two proofs do not require the hypothesis. We simply recall them here without proofs.

Lemma 3.4. [30, Lemma 4.3] We use the notation in Notation [3.1. Suppose $(u=$ $\left.p_{0}\right) p_{1} \cdots p_{2 k}\left(p_{2 k+1}=v\right)$ is an even-connection in $G$ with respect to $e_{1} \cdots e_{s}$ for some $k \geq 1$. If $\left\{w, p_{i}\right\} \in E\left(G^{\prime}\right)$ for some $0 \leq i \leq 2 k+1$, then either $\{u, w\} \in E\left(G^{\prime}\right)$ or $\{v, w\} \in E\left(G^{\prime}\right)$.

Lemma 3.5. [30, Lemma 4.5] Let the notation be as in Notation [3.1. Let $y \in V(G)$ and $H=G \backslash N_{G}[y]$. If $\left\{e_{1}, \ldots, e_{s}\right\} \cap E(H)=\left\{e_{i_{1}}, \ldots, e_{i_{t}}\right\}$ and $H^{\prime}$ is the graph associated to $\left(I(H)^{t+1}: e_{i_{1}} \cdots e_{i_{t}}\right)$, then $G^{\prime} \backslash N_{G^{\prime}}[y]$ is an induced subgraph of $H^{\prime}$. In particular,

$$
\operatorname{reg}\left(I\left(G^{\prime} \backslash N_{G^{\prime}}[y]\right)\right) \leq \operatorname{reg}\left(I\left(H^{\prime}\right)\right) .
$$

In the following results, we show that the even-connections in a parent graph with respect to edges coming from an induced subgraph, induces an even-connection in the induced subgraph.

Lemma 3.6. Let $G$ be a graph and $H$ be an induced subgraph of $G$. For $e_{1}, \ldots, e_{s} \in E(H)$, $s \geq 1$, let $H^{\prime}$ and $G^{\prime}$ be the graphs associated to $\left(I(H)^{s+1}: e_{1} \cdots e_{s}\right)$ and $\left(I(G)^{s+1}: e_{1} \cdots e_{s}\right)$ respectively. Then $H^{\prime}$ is an induced subgraph of $G^{\prime}$. In particular,

$$
\operatorname{reg}\left(I\left(H^{\prime}\right)\right) \leq \operatorname{reg}\left(I\left(G^{\prime}\right)\right) \text {. }
$$

Proof. Let $a, b \in V(H)$, not necessarily distinct, be such that $a$ is even-connected to $b$ in $H$ with respect to $e_{1} \cdots e_{s}$. For some $k \geq 0$, let $\left(a=p_{0}\right) p_{1} \cdots p_{2 k}\left(p_{2 k+1}=b\right)$ be an even-connection in $H$. Since $H$ is an induced subgraph of $G,\left(a=p_{0}\right) p_{1} \cdots p_{2 k}\left(p_{2 k+1}=b\right)$ 
is an even-connection in $G$ with respect to $e_{1} \cdots e_{s}$. Therefore $a$ is even-connected to $b$ in $G$ with respect to $e_{1} \cdots e_{s}$. Hence $H^{\prime}$ is a subgraph of $G^{\prime}$. Since $H$ is an induced subgraph of $G$ and $e_{1}, \ldots, e_{s} \in E(H)$, any even-connection between vertices of $V(H)$ in $G$ with respect to $e_{1} \cdots e_{s}$ is an even-connection in $H$ as well. Hence $H^{\prime}$ is an induced subgraph of $G^{\prime}$. The assertion on the regularity follows from [28, Proposition 4.1.1].

Let the notation be as in Notation 3.1. For some $1 \leq \alpha \leq s$, set $e_{\alpha}=\{x, y\}$. We further explore the even-connections between $N_{G^{\prime}}[y]$ and $N_{G^{\prime}}(x)$. If $\left(u=p_{0}\right) p_{1} \cdots p_{2 k}\left(p_{2 k+1}=y\right)$ ( $u$ may be equal to $y$ ) is an even-connection in $G$ with respect to $e_{1} \cdots e_{s}$, then there are four possibilities:

(i) $\{u, y\} \in E(G)$ i.e., $k=0$;

(ii) $\left\{p_{2 \lambda+1}, p_{2 \lambda+2}\right\} \neq e_{\alpha}$ for any $0 \leq \lambda \leq k-1$;

(iii) There exists $0 \leq \lambda \leq k-1$ with $\left\{p_{2 \lambda+1}, p_{2 \lambda+2}\right\}=e_{\alpha}$ and $p_{2 \lambda+1}=y, p_{2 \lambda+2}=x$;

(iv) There exists $0 \leq \lambda \leq k-1$ with $\left\{p_{2 \lambda+1}, p_{2 \lambda+2}\right\}=e_{\alpha}$, and for all such $\lambda$, we have $p_{2 \lambda+1}=x$ and $p_{2 \lambda+2}=y$.

Let

$$
\begin{gathered}
\mathcal{X}_{y}=\left\{u \in V(G) \mid \text { there exists an even-connection }\left(u=p_{0}\right) p_{1} \cdots p_{2 k}\left(p_{2 k+1}=y\right)\right. \\
\text { satisfies (i), (ii) or (iii) }\} .
\end{gathered}
$$

Note that $N_{G}(y) \subseteq \mathcal{X}_{y}$. It may also be noted that if $u \in N_{G^{\prime}}(y) \backslash \mathcal{X}_{y}$, then for any even-connection $\left(u=p_{0}\right) p_{1} \cdots p_{2 k}\left(p_{2 k+1}=y\right)$, the conditions (i), (ii) and (iii) are not satisfied.

We illustrate the definition of $\mathcal{X}_{y}$ with an example below. Let $G$ be the graph as shown in the figure below. Let $e_{1}=\{x, y\}, e_{2}=\left\{x_{3}, x_{4}\right\}, e_{3}=\left\{x_{5}, x_{6}\right\}, e_{4}=\left\{x_{7}, x_{8}\right\}$ and let $G^{\prime}$ be the graph associated to $\left(I(G)^{5}: e_{1} e_{2} e_{3} e_{4}\right)$.

Then $x_{9} x_{8} x_{7} y$ and $y x_{6} x_{5} y$ are even-connections in $G$ with respect

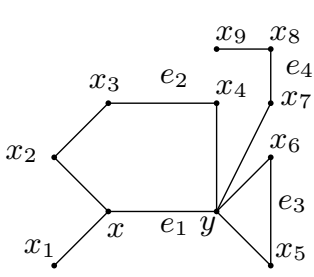
to $e_{1} e_{2} e_{3} e_{4}$. Both even-connections satisfy (ii). Hence $x_{9}, y \in \mathcal{X}_{y}$. The even-connection, $x_{2} x y x_{6} x_{5} y$, with respect to $e_{1} e_{2} e_{3} e_{4}$ does not satisfy, (i), (ii) and (iii). At the same time, $x_{2} x_{3} x_{4} y$ is an evenconnection with respect to $e_{1} e_{2} e_{3} e_{4}$ and it satisfies (ii). Therefore $x_{2} \in \mathcal{X}_{y}$. Hence $\mathcal{X}_{y}=\left\{x, x_{4}, x_{6}, x_{5}, x_{7}, x_{9}, y, x_{2}\right\}$. It can also be noted that $x_{1} \notin \mathcal{X}_{y}$.

The following lemma will play a crucial role in the study of the regularity of powers of edge ideals in the next section.

Lemma 3.7. Let the notation be as above. For some $1 \leq \alpha \leq s$, set $e_{\alpha}=\{x, y\}$.

(1) If $N_{G^{\prime}}(y) \backslash \mathcal{X}_{y} \neq \emptyset$, then $y \in \mathcal{X}_{y}$.

(2) If $y$ is even-connected to itself, then $y \in \mathcal{X}_{y}$.

(3) If $u \in \mathcal{X}_{y}$, then $G^{\prime} \backslash N_{G^{\prime}}[u]$ is an induced subgraph of $\left(G \backslash N_{G}[u, x]\right)^{\prime}$, where $(G \backslash$ $\left.N_{G}[u, x]\right)^{\prime}$ is the graph associated to $\left(I\left(G \backslash N_{G}[u, x]\right)^{t+1}: e_{j_{1}} \cdots e_{j_{t}}\right)$ and $\left\{e_{j_{1}}, \ldots, e_{j_{t}}\right\}=$ $E\left(G \backslash N_{G}[u, x]\right) \cap\left\{e_{1}, \ldots, e_{s}\right\}$. In particular,

$$
\operatorname{reg}\left(I\left(G^{\prime} \backslash N_{G^{\prime}}[u]\right)\right) \leq \operatorname{reg}\left(I\left(\left(G \backslash N_{G}[u, x]\right)^{\prime}\right)\right) .
$$


(4) The graph $G^{\prime} \backslash \mathcal{X}_{y}$ is an induced subgraph of $\left(G \backslash N_{G}[y]\right)^{\prime}$, where $\left(G \backslash N_{G}[y]\right)^{\prime}$ is the graph associated to $\left(I\left(G \backslash N_{G}\left[\overline{y])^{t+1}}: e_{j_{1}} \cdots e_{j_{t}}\right)\right.\right.$ and $\left\{e_{j_{1}}, \ldots, e_{j_{t}}\right\}=E(G \backslash$ $\left.N_{G}[y]\right) \cap\left\{e_{1}, \ldots, e_{s}\right\}$. In particular,

$$
\operatorname{reg}\left(I\left(G^{\prime} \backslash \mathcal{X}_{y}\right)\right) \leq \operatorname{reg}\left(I\left(\left(G \backslash N_{G}[y]\right)^{\prime}\right)\right) .
$$

Proof. (1) Let $u \in N_{G^{\prime}}(y) \backslash \mathcal{X}_{y}$ and $\left(u=p_{0}\right) p_{1} \cdots\left(p_{2 k+1}=y\right)$ be an even-connection in $G$ with respect to $e_{1} \cdots e_{s}$. Since $u \notin \mathcal{X}_{y}$, there exists $0 \leq \lambda \leq k-1$ with $\left\{p_{2 \lambda+1}, p_{2 \lambda+2}\right\}=e_{\alpha}$ with $p_{2 \lambda+1}=x$ and $p_{2 \lambda+2}=y$. Let $\gamma$ be the largest integer with this property. Note that $p_{2 \gamma+2}=y$. Then $\left(y=p_{2 \gamma+2}\right) p_{2 \gamma+3} \cdots p_{2 k}\left(p_{2 k+1}=y\right)$ is an even-connection in $G$ and $\left\{p_{2 \lambda^{\prime}+1}, p_{2 \lambda^{\prime}+2}\right\} \neq e_{\alpha}$ for all $\gamma+1 \leq \lambda^{\prime} \leq k-1$. Therefore $y \in \mathcal{X}_{y}$.

(2) Assume, in contrary, that any even-connection from $y$ to $y$ with respect to $e_{1} \cdots e_{s}$ does not satisfy (ii) and (iii). Then by taking $u=y$ in the proof of (1), it can be seen that there exists an even-connection from $y$ to itself which satisfy the condition (ii). Hence $y \in \mathcal{X}_{y}$.

(3) Set $H=G^{\prime} \backslash N_{G^{\prime}}[u]$ and $K=\left(G \backslash N_{G}[u, x]\right)^{\prime}$. Let $a, b \in V(H)$, not necessarily distinct, be such that $a$ is even-connected to $b$ in $G$ with respect to $e_{1} \cdots e_{s}$. Let $(a=$ $\left.q_{0}\right) q_{1} \cdots\left(q_{2 l+1}=b\right)$ be an even-connection in $G$ with respect to $e_{1} \cdots e_{s}$. We claim that $q_{r} \notin N_{G}[u, x]$ for all $0 \leq r \leq 2 l+1$. Note that by Lemma [3.4, $q_{r} \notin N_{G^{\prime}}[u]$ for all $0 \leq r \leq 2 l+1$. Suppose $q_{r} \in N_{G}[x]$ for some $0 \leq r \leq 2 l+1$. Since $u \in \mathcal{X}_{y}, u$ is even-connected to $q_{r}$ in $G$ with respect to $e_{1} \cdots e_{s}$. By Lemma 3.4, $u$ is even-connected either to $a$ or to $b$ in $G$ with respect to $e_{1} \cdots e_{s}$. Hence either $a$ or $b$ belongs to $N_{G^{\prime}}[u]$. This is a contradiction to our assumption that $a, b \in V(H)$. Hence $q_{r} \notin N_{G}[x]$ for all $0 \leq r \leq 2 l+1$. Therefore $a$ is even-connected to $b$ in $G \backslash N_{G}[u, x]$ with respect to $e_{j_{1}} \cdots e_{j_{t}}$.

(4) Let $a, b \in V\left(G^{\prime} \backslash \mathcal{X}_{y}\right)$ and $a$ be even-connected to $b$ in $G$ with respect to $e_{1} \cdots e_{s}$. Using the fact that $N_{G}(y) \subseteq \mathcal{X}_{y}$ and by (1) and (2), we get $a, b \notin N_{G}[y]$. Hence, if $\{a, b\} \in E(G)$, then $\{a, b\} \in E\left(G \backslash N_{G}[y]\right)$. Let $\left(a=q_{0}\right) q_{1} \cdots\left(q_{2 l+1}=b\right)$ be an even-connection in $G$ with respect to $e_{1} \cdots e_{s}$. We claim that $q_{j} \notin N_{G}[y]$ for any $j$. Suppose $q_{j} \in N_{G}(y)$ for some $0 \leq j \leq 2 l+1$. If $j$ is odd, then choose the largest integer $r$ such that $q_{r} \in N_{G}(y)$. Then $\left(b=q_{2 l+1}\right) q_{2 l} \cdots q_{r} y$ is an even-connection in $G$ with respect to $e_{1} \cdots e_{s}$. Since $r$ is the largest integer, $q_{j} \notin N_{G}[y]$ for all $r<j \leq 2 l+1$. Therefore $b \in \mathcal{X}_{y}$ which is a contradiction. Now if $r$ is even, then choose the smallest integer $r$ such that $q_{r} \in N_{G}(y)$. Then $\left(a=q_{0}\right) q_{1} \cdots q_{r} y$ is an even-connection in $G$ with respect to $e_{1} \cdots e_{s}$. Therefore $a \in \mathcal{X}_{y}$ which again is a contradiction. Hence $q_{j} \notin N_{G}(y)$ for all $j$. If $q_{j}=y$ for some $0 \leq j \leq 2 l+1$, then $1 \leq j \leq 2 l$ either $q_{j-1} \in N_{G}(y)$ or $q_{j+1} \in N_{G}(y)$ which contradicts the first part of the proof. This completes the proof of the claim. This shows that $a$ is even-connected to $b$ in $G \backslash N_{G}[y]$ with respect to $e_{j_{1}} \cdots e_{j_{t}}$.

As in Lemma 3.6, it can be seen that the subgraphs considered in (3) and (4) are induced subgraphs. The assertion on the regularity in (3) and (4) follows from [28, Proposition 4.1.1].

\section{Regularity of POWERS OF GRAPHS}

In this section, we obtain a general upper bound for the regularity of powers of edge ideals of graphs. The first main theorem gives certain sufficient conditions for any combinatorial invariant to be an upper bound for the constant term of the linear polynomial 
corresponding to $\operatorname{reg}\left(I(G)^{q}\right)$. The below result can be seen as a different version of 4 , Theorem 3.3].

Theorem 4.1. Let $G$ be a graph and $\rho: \mathcal{I}_{G} \longrightarrow \mathbb{N}$ be a function such that for any $L \in \mathcal{I}_{G}$,

(1) $\operatorname{reg}(I(L)) \leq \rho(L)+1$,

(2) $\rho\left(L_{1}\right) \leq \rho(L)$ for any induced subgraph $L_{1}$ of $L$ and

(3) there exists a vertex $x \in V(L)$ such that $\rho\left(L \backslash N_{L}[x]\right)+1 \leq \rho(L)$.

Then

$$
\operatorname{reg}\left(I(G)^{q}\right) \leq 2 q+\rho(G)-1 \text { for all } q \geq 1
$$

Proof. Let $G$ be a graph and $\rho: \mathcal{I}_{G} \longrightarrow \mathbb{N}$ be a function satisfying the given hypotheses. We prove the assertion by induction on $q$. The case $q=1$ follows from the assumption. Assume that $q>1$. For any graph $K$, set

$$
\mathcal{P}(K)=\left\{x \in V(K) \mid \rho(K) \geq \rho\left(K \backslash N_{K}[x]\right)+1\right\} .
$$

By hypothesis, $\mathcal{P}(G) \neq \emptyset$. By applying [2, Theorem 5.2] and using induction, it is enough to prove that for edges $e_{1}, \ldots, e_{s}$ of $G, \operatorname{reg}\left(\left(I(G)^{s+1}: e_{1} \cdots e_{s}\right)\right) \leq \rho(G)+1$ for all $s \geq 0$. Let $G^{\prime}$ be the graph associated to the ideal $\left(I(G)^{s+1}: e_{1} \cdots e_{s}\right)$ which is contained in an appropriate polynomial ring $R_{1}$. We prove that $\operatorname{reg}\left(I\left(G^{\prime}\right)\right) \leq \rho(G)+1$ by induction on $s+|V(G)|$.

If $s=0$, then $G^{\prime}=G$ and hence the assertion is true for any value of $|V(G)|$. Therefore, we may assume that $s \geq 1$. If $|V(G)|=2$, then $G$ consists of only one edge. In this case, we also have $G^{\prime}=G$ and hence the assertion is true. Now, assume that $s \geq 1$ and $|V(G)|>2$.

Let $e_{i}=\left\{a_{i}, b_{i}\right\}$ for $1 \leq i \leq s$. If $\operatorname{deg}_{G}\left(a_{i}\right)=1$ or $\operatorname{deg}_{G}\left(b_{i}\right)=1$ for some $i$, then by Lemma 3.2 , it follows that

$$
\operatorname{reg}\left(I(G)^{s+1}: e_{1} \cdots e_{s}\right)=\operatorname{reg}\left(I(G)^{s}: e_{1} \cdots e_{i-1} e_{i+1} \cdots e_{s}\right) \leq \rho(G)+1,
$$

where the last inequality follows from the hypothesis of induction.

Assume now that $\operatorname{deg}_{G}\left(a_{i}\right) \geq 2$ and $\operatorname{deg}_{G}\left(b_{i}\right) \geq 2$ for all $1 \leq i \leq s$.

CASE 1: Suppose $e_{i} \cap \mathcal{P}(G) \neq \emptyset$ for some $1 \leq i \leq s$.

Without loss of generality, we may assume that $e_{s} \cap \mathcal{P}(G) \neq \emptyset$ and $a_{s} \in \mathcal{P}(G)$. Let $J=I\left(G^{\prime}\right)$. Following the notation as in (3.1), set $\mathcal{X}_{b_{s}}=\left\{y_{1}, \ldots, y_{p}\right\}$. It follows from the collection of short exact sequences:

$$
\begin{aligned}
0 & \longrightarrow \frac{R_{1}}{\left(J: y_{1}\right)}(-1) \stackrel{\cdot y_{1}}{\longrightarrow} \frac{R_{1}}{J} \longrightarrow \frac{R_{1}}{J+\left(y_{1}\right)} \longrightarrow 0 ; \\
\vdots & \vdots \\
0 & \longrightarrow \frac{R_{1}}{\left(\left(J+\left(y_{1}, \ldots, y_{p-1}\right)\right): y_{p}\right)}(-1) \stackrel{\cdot y_{p}}{\longrightarrow} \frac{R_{1}}{J+\left(y_{1}, \ldots, y_{p-1}\right)} \longrightarrow \frac{R_{1}}{J+\left(\mathcal{X}_{b_{s}}\right)} \longrightarrow 0,
\end{aligned}
$$

that

$$
\operatorname{reg}\left(R_{1} / J\right) \leq \max \left\{\operatorname{reg}\left(\frac{R_{1}}{\left(J: y_{1}\right)}\right)+1, \ldots, \operatorname{reg}\left(\frac{R_{1}}{\left(\left(J+\left(y_{1}, \ldots, y_{p-1}\right)\right): y_{p}\right)}\right)+1, \operatorname{reg}\left(\frac{R_{1}}{J+\left(\mathcal{X}_{b_{s}}\right)}\right)\right\} .
$$


Now,

$$
\begin{aligned}
\operatorname{reg}\left(J+\left(\mathcal{X}_{b_{s}}\right)\right) & =\operatorname{reg}\left(I\left(G^{\prime} \backslash \mathcal{X}_{b_{s}}\right)\right) & & \text { (by [8, Remark 2.5]) } \\
& \leq \operatorname{reg}\left(I\left(\left(G \backslash N_{G}\left[b_{s}\right]\right)^{\prime}\right)\right), & & \text { (by Lemma [3.7] }(4))
\end{aligned}
$$

where $E\left(G \backslash N_{G}\left[b_{s}\right]\right) \cap\left\{e_{1}, \ldots, e_{s}\right\}=\left\{e_{j_{1}}, \ldots, e_{j_{t}}\right\}$ and $\left(G \backslash N_{G}\left[b_{s}\right]\right)^{\prime}$ is the graph associated to $\left(I\left(G \backslash N_{G}\left[\widehat{\left.\left.b_{s}\right]\right)^{t+1}}: e_{j_{1}} \cdots e_{j_{t}}\right)\right.\right.$. Therefore

$$
\operatorname{reg}\left(J+\left(\mathcal{X}_{b_{s}}\right)\right) \leq \operatorname{reg}\left(I\left(\left(G \backslash N_{G}\left[b_{s}\right]\right)^{\prime}\right)\right) \leq \rho\left(G \backslash N_{G}\left[b_{s}\right]\right)+1 \leq \rho(G)+1,
$$

where the second and last inequalities follows by induction on the number of vertices and the assumption (2) respectively. Using similar arguments, we get

$$
\begin{aligned}
\operatorname{reg}\left(\left(J: y_{i}\right)\right)=\operatorname{reg}\left(I\left(G^{\prime} \backslash N_{G^{\prime}}\left[y_{i}\right]\right)\right) & \leq \operatorname{reg}\left(I\left(\left(G \backslash N_{G}\left[y_{i}, a_{s}\right]\right)^{\prime}\right)\right) & & \text { (by Lemma } 3.7(3)) \\
& \leq \operatorname{reg}\left(I\left(\left(G \backslash N_{G}\left[a_{s}\right]\right)^{\prime}\right)\right) & & \text { (by Lemma [3.6) } \\
& \leq \rho\left(G \backslash N_{G}\left[a_{s}\right]\right)+1 & & \text { (by induction) } \\
& <\rho(G)+1, & &
\end{aligned}
$$

where the last inequality follows by the assumption that $a_{s} \in \mathcal{P}(G)$.

Note that $\left(\left(J+\left(y_{1}, \ldots, y_{i-1}\right)\right): y_{i}\right)$ is the edge ideal of $\left(G^{\prime} \backslash N_{G^{\prime}}\left[y_{i}\right]\right) \backslash\left\{y_{1}, \ldots, y_{i-1}\right\}$ and $\left(J: y_{i}\right)$ is the edge ideal of $G^{\prime} \backslash N_{G^{\prime}}\left[y_{i}\right]$. Since $\left(G^{\prime} \backslash N_{G^{\prime}}\left[y_{i}\right]\right) \backslash\left\{y_{1}, \ldots, y_{i-1}\right\}$ is an induced subgraph of $G^{\prime} \backslash N_{G^{\prime}}\left[y_{i}\right]$, it follows that

$$
\operatorname{reg}\left(\left(\left(J+\left(y_{1}, \ldots, y_{i-1}\right)\right): y_{i}\right)\right) \leq \operatorname{reg}\left(J: y_{i}\right)<\rho(G)+1 .
$$

Therefore $\operatorname{reg}(J) \leq \rho(G)+1$.

CAse 2: Suppose $e_{i} \cap \mathcal{P}(G)=\emptyset$ for all $1 \leq i \leq s$. Let $x \in \mathcal{P}(G)$. Then by [21, Theorem $3.4]$

$$
\operatorname{reg}\left(I\left(G^{\prime}\right)\right) \leq \max \left\{\operatorname{reg}\left(I\left(G^{\prime} \backslash x\right)\right), \operatorname{reg}\left(I\left(G^{\prime} \backslash N_{G^{\prime}}[x]\right)\right)+1\right\} .
$$

By Lemma 3.3 and inductive hypothesis we get

$$
\left.\operatorname{reg}\left(I\left(G^{\prime} \backslash x\right)\right)=\operatorname{reg}\left(I(G \backslash x)^{s+1}: e_{1} \cdots e_{s}\right)\right) \leq \rho(G \backslash x)+1 \leq \rho(G)+1 .
$$

Similarly, by Lemma 3.5 and inductive hypothesis we get

$$
\operatorname{reg}\left(I\left(G^{\prime} \backslash N_{G^{\prime}}[x]\right)\right) \leq \operatorname{reg}\left(I\left(G \backslash N_{G}[x]\right)^{t+1}: e_{i_{1}} \cdots e_{i_{t}}\right) \leq \rho\left(G \backslash N_{G}[x]\right)+1 \leq \rho(G),
$$

where the last inequality follows by the assumption that $x \in \mathcal{P}(G)$, and $\left\{e_{i_{1}}, \ldots, e_{i_{t}}\right\}=$ $E\left(G \backslash N_{G}[x]\right) \cap\left\{e_{1}, \ldots, e_{s}\right\}$. Therefore $\operatorname{reg}\left(I\left(G^{\prime}\right)\right) \leq \rho(G)+1$.

This completes the proof.

As a consequence of Theorem 4.1, we obtain a sufficient condition for the Conjecture 1.2 to be true.

Corollary 4.2. Let $G$ be a graph. If every non-empty induced subgraph $H$ of $G$ has a vertex $x$ with $\operatorname{reg}\left(I\left(H \backslash N_{H}[x]\right)\right)+1 \leq \operatorname{reg}(I(H))$, then for all $q \geq 1$,

$$
\operatorname{reg}\left(I(G)^{q}\right) \leq 2 q+\operatorname{reg}(I(G))-2 .
$$

Proof. For $L \in \mathcal{I}_{G}$, let $\rho(L)=\operatorname{reg}(I(L))-1$. Then it is easy to see that $\rho$ satisfies $(1)$ (3) of Theorem 4.1. Hence the assertion follows. 
It is interesting to ask if every graph $G$ has a vertex $x$ with $\operatorname{reg}\left(I\left(G \backslash N_{G}[x]\right)\right)+1 \leq$ $\operatorname{reg}(I(G))$. It was communicated to us by Tran Nam Trung that there exists a graph $G$ which does not satisfy the hypothesis of Corollary 4.2. Let $G$ be the graph denoted by $G_{2}$ in Appendix A of [31, page 452. Then for every vertex $x$ of $G$, it can be verified that $\operatorname{reg}(I(G))=\operatorname{reg}\left(I\left(G \backslash N_{G}[x]\right)\right)$. Hence we would like to ask:

Question 4.3. Can we classify graphs $G$ having a vertex $x$ such that $\operatorname{reg}\left(I\left(G \backslash N_{G}[x]\right)\right)+$ $1 \leq \operatorname{reg}(I(G))$ ?

As more applications of Theorem 4.1, we obtain upper bounds for the regularity of powers of edge ideals. We first recall the definitions of the invariants co-chord $(G)$ and $\zeta(G)$.

The complement of a graph $G$, denoted by $G^{c}$, is the graph on the same vertex set as $G$ in which $\{u, v\}$ is an edge of $G^{c}$ if and only if it is not an edge of $G$. A graph $G$ is chordal if every induced cycle in $G$ has length 3, and is co-chordal if the complement graph $G^{c}$ is chordal. The co-chordal cover number, denoted co-chord $(G)$, is the minimum number $n$ such that there exist co-chordal subgraphs $H_{1}, \ldots, H_{n}$ of $G$ with $E(G)=\bigcup_{i=1}^{n} E\left(H_{i}\right)$.

Now we recall the definition of $\zeta(G)$ from [23]. A star at $x$, which is the subgraph on $N_{G}[x]$ with edge set consisting of all edges of $G$ incident to $x$. We say that a star is nondegenerate if $\operatorname{deg}_{G}(x)>1$, so that the star does not consist of a single vertex or a single edge. We say a set of stars is center-separated if the center of a star and at least two of its neighbors are not contained in any other star. In the collection of all stars in $G$, a maximal center-separated star packing of $G$ is a center-separated star packing of $G$ that is not a subset of any other center-separated star packing. Let $\mathcal{P}$ be a maximal centerseparated star packing. After deleting the vertices of the stars $\mathcal{P}$, an induced matching of $G$ will remain. Let $\zeta_{\mathcal{P}}$ be the number of stars in the packing plus the number of edges in the remained induced matching and let $\zeta(G)$ be the maximum $\zeta_{\mathcal{P}}$ over all maximal center-separated packings of nondegenerate stars.

For example, if $G=C_{n}$, cycle on $\left\{x_{1}, \ldots, x_{n}\right\}$ vertices, then for any $x \in V(G)$, star at $x$ is a path on 3 vertices and hence $G \backslash N_{G}[x]$ is a path on $n-3$ vertices. If $n \equiv 0,1(\bmod 3)$, then $\mathcal{P}=\left\{\right.$ star at $x_{1}$, star at $x_{4}$, star at $x_{7}, \ldots$, star at $\left.x_{n-2}\right\}$ is a center-separated star packing of $G$. Therefore $\zeta_{\mathcal{P}}=\left\lfloor\frac{n}{3}\right\rfloor$. If $n \equiv 2(\bmod 3)$, then $\mathcal{P}^{\prime}=\left\{\right.$ star at $x_{1}$, star at $x_{4}, \ldots$, star at $\left.x_{n-4}\right\}$ is a center separated star packing of $G$. Note that $G \backslash \mathcal{P}^{\prime}$ consists of a single edge. Hence $\zeta_{\mathcal{P}^{\prime}}=\left\lfloor\frac{n}{3}\right\rfloor+1$. Therefore, if $n \equiv 0,1(\bmod 3)$, then $\zeta(G) \geq\left\lfloor\frac{n}{3}\right\rfloor$ and if $n \equiv 2(\bmod 3)$, then $\zeta(G) \geq\left\lfloor\frac{n}{3}\right\rfloor+1$. It is not hard to verify that the above inequalities are in fact equalities. Also, note that co-chordal graphs do not have two disjoint edges. Therefore, we have:

(1) if $n \equiv 0(\bmod 3)$ or $n=4$, then $\nu\left(C_{n}\right)=\operatorname{co-chord}\left(C_{n}\right)=\zeta\left(C_{n}\right)=\left\lfloor\frac{n}{3}\right\rfloor$;

(2) if $n \equiv 1(\bmod 3)$ and $n>4$, then $\nu\left(C_{n}\right)=\zeta\left(C_{n}\right)=\operatorname{co-chord}\left(C_{n}\right)-1=\left\lfloor\frac{n}{3}\right\rfloor$;

(3) if $n \equiv 2(\bmod 3)$, then $\nu\left(C_{n}\right)=\zeta\left(C_{n}\right)-1=\operatorname{co-} \operatorname{chord}\left(C_{n}\right)-1=\left\lfloor\frac{n}{3}\right\rfloor$.

It may be noted that for any graph $G, \nu(G) \leq \zeta(G)$. Hà and Woodroofe proved that for a graph $G, \operatorname{reg}(I(G)) \leq \zeta(G)+1$, [23]. Also, it was proved by Woodroofe that $\operatorname{reg}(I(G)) \leq \operatorname{co-chord}(G)+1$, [45, Theorem 1]. We would like to note here that the invariants $\zeta(G)$ and co-chord $(G)$ are not comparable in general. For example, if $G=C_{7}$, then $\operatorname{co-chord}(G)=3$ and $\zeta(G)=2$. If $H$ is the graph with $E(H)=$ 
$\left\{\left\{x_{1}, x_{2}\right\},\left\{x_{2}, x_{3}\right\},\left\{x_{3}, x_{4}\right\},\left\{x_{4}, x_{1}\right\},\left\{x_{1}, x_{5}\right\}\right\}$, then it is easy to see that co-chord $(H)=1$ and $\zeta(H)=2$. Now we prove one of the main results of this paper.

Theorem 4.4. Let $G$ be a graph. Then for all $q \geq 1$,

(1) $\operatorname{reg}\left(I(G)^{q}\right) \leq 2 q+\zeta(G)-1$.

(2) $\operatorname{reg}\left(I(G)^{q}\right) \leq 2 q+$ co-chord $(G)-1$.

Proof. From [23, Theorem 1.6] and [45, Theorem 1], it follows that for any graph $G$, $\operatorname{reg}(I(G)) \leq \zeta(G)+1$ and $\operatorname{reg}(I(G)) \leq$ co-chord $(G)+1$. It is easy to see that, for any induced subgraph $L$ of $G, \zeta(L) \leq \zeta(G)$ and co-chord $(L) \leq \operatorname{co-chord}(G)$.

(1) Suppose that there exists a vertex $x$ in $G$ such that $\operatorname{deg}_{G}(x) \geq 2$. Let $H=G \backslash N_{G}[x]$. Let $\mathcal{P}^{\prime}$ be a maximal center-separated star packing of $H$ such that $\zeta_{\mathcal{P}^{\prime}}(H)=\zeta(H)$. Then $\mathcal{P}=\mathcal{P}^{\prime} \cup\{$ star at $x\}$ is a center-separated star packing of $G$. Thus, $\zeta(H)+1=\zeta_{\mathcal{P}^{\prime}}(H)+$ $1=\zeta_{\mathcal{P}}(G) \leq \zeta(G)$. If $\operatorname{deg}_{G}(x)=1$ for all $x \in V(G)$, then by definition $\zeta\left(G \backslash N_{G}[x]\right)+1 \leq$ $\zeta(G)$ for all $x \in V(G)$. Therefore, by Theorem 4.1, $\operatorname{reg}\left(I(G)^{q}\right) \leq 2 q+\zeta(G)-1$.

(2) It follows from [38, Lemma 3.1] that there is a vertex $x \in V(G)$ such that co-chord $(G \backslash$ $\left.N_{G}[x]\right)+1 \leq \operatorname{co-chord}(G)$. Hence, by Theorem 4.1, $\operatorname{reg}\left(I(G)^{q}\right) \leq 2 q+\operatorname{co-chord}(G)-1$.

A graph $G$ is said to be weakly chordal if neither $G$ nor $G^{c}$ contain induced cycle of length 5 or more. It is straightforward to show that a chordal graph is weakly chordal. The matching number of $G$, denoted by $\mathrm{m}(G)$, is the maximum cardinality among matchings of $G$ and the minimum matching number of $G$, denoted by $\min -\max (G)$, is the minimum cardinality among maximal matchings of $G$. By [27, p. 2], [45, Theorem 1], [29, p. 10], for any graph $G$, we have

$$
\nu(G) \leq \operatorname{co-chord}(G) \leq \min -\max (G) \leq \mathrm{m}(G) .
$$

Many authors have studied classes of graphs whose induced matching number coincides with co-chord $(G), \min -\max (G)$ or $\mathrm{m}(G)$. For example, it is known that $\nu(G)=$ co-chord $(G)$ for unmixed bipartite graphs ([45, Theorem 16]), weakly chordal graphs ([12, Proposition 3]) and bipartite graphs with $\operatorname{reg}(I(G))=3([29$, Observation 5.3]). For Cameron-Walker graphs $\nu(G)=\mathrm{m}(G),([13,26])$. Hibi et al. studied the class of graphs for which $\nu(G)=\min -\max (G)$, [27]. Beyarslan, Hà and Trung proved that $\operatorname{reg}\left(I(G)^{q}\right) \geq$ $2 q+\nu(G)-1$ for any graph $G$ and for all $q \geq 1$, [8, Theorem 4.5]. Hence, we can use Theorem 4.4 for the above mentioned classes of graphs to get $\operatorname{reg}\left(I(G)^{q}\right)=2 q+\nu(G)-1$ for all $q \geq 1$.

\section{Corollary 4.5. The following hold:}

(1) If $G$ is a weakly chordal graph, then $\operatorname{reg}\left(I(G)^{q}\right)=2 q+\nu(G)-1$ for all $q \geq 1$.

(2) If $G$ is a Cameron-Walker graph, then $\operatorname{reg}\left(I(G)^{q}\right)=2 q+\nu(G)-1$ for all $q \geq 1$.

(3) [25, Theorem 3.2] If $I(G)$ has a linear resolution (i.e., $G^{c}$ is chordal), then $I(G)^{q}$ has a linear resolution for all $q \geq 2$.

(4) [8, Theorem 4.7] If $G$ is a forest, then $\operatorname{reg}\left(I(G)^{q}\right)=2 q+\nu(G)-1$ for all $q \geq 1$.

(5) [29, Corollary 5.1(1)] If $G$ is an unmixed bipartite graph, then $\operatorname{reg}\left(I(G)^{q}\right)=2 q+$ $\nu(G)-1$ for all $q \geq 1$.

(6) [1, Theorem 3.9] If $G$ is a bipartite graph and $\operatorname{reg}(I(G))=3$, then $\operatorname{reg}\left(I(G)^{q}\right)=$ $2 q+1$ for all $q \geq 1$. 
For two vertex disjoint graphs $G_{1}$ and $G_{2}$, we denote the union of $G_{1}$ and $G_{2}$ by $G_{1} \coprod G_{2}$ i.e., $V\left(G_{1} \coprod G_{2}\right)=V\left(G_{1}\right) \cup V\left(G_{2}\right)$ and $E\left(G_{1} \coprod G_{2}\right)=E\left(G_{1}\right) \cup E\left(G_{2}\right)$. Note that $\Psi\left(G_{1} \coprod G_{2}\right)=\Psi\left(G_{1}\right)+\Psi\left(G_{2}\right)$ whenever $\Psi(-)$ is equal to $\nu(-)$, co-chord $(-)$, min-max $(-)$, $\mathrm{m}(-)$ or $\zeta(-)$. If $G_{1}$ and $G_{2}$ are graphs for which the linear polynomials corresponding to $\operatorname{reg}\left(I\left(G_{1}\right)^{q}\right)$ and $\operatorname{reg}\left(I\left(G_{2}\right)^{q}\right)$ are known, then using [35, Theorem 5.7] it is possible to compute the linear polynomial corresponding to $\operatorname{reg}\left(\left(I\left(G_{1}\right)+I\left(G_{2}\right)\right)^{q}\right)=\operatorname{reg}\left(I\left(G_{1} \coprod G_{2}\right)^{q}\right)$.

Proposition 4.6. Let $G_{1}=\left(\coprod_{i=1}^{t} C_{n_{i}}\right)$ where $t \geq 1$ and $n_{1}, \ldots, n_{t} \equiv 2(\bmod 3)$. Let $G_{2}$ be an arbitrary graph and set $G=G_{1} \amalg G_{2}$.

(1) Then $\operatorname{reg}\left(I\left(G_{1}\right)^{q}\right)=2 q+\nu\left(G_{1}\right)+t-2$ for all $q \geq 2$.

(2) If $\operatorname{reg}\left(I\left(G_{2}\right)^{q}\right)=2 q+\nu\left(G_{2}\right)-1$ for all $q \geq 1$, then $\operatorname{reg}\left(I(G)^{q}\right)=2 q+\nu(G)+t-1$ for all $q \geq 2$.

Proof. By [45, Lemma 8], [28, Theorem 7.6.28], $\operatorname{reg}\left(I\left(G_{1}\right)\right)=\nu\left(G_{1}\right)+t+1$.

(1) We prove by induction on $t$. If $t=1$, then the assertion follows from [8, Theorem 5.2]. Set $H=\coprod_{i=1}^{t-1} C_{n_{i}}$. Also, from [45, Lemma 8], we get $\operatorname{reg}(I(H))=\nu(H)+t$. By inductive hypothesis on $t, \operatorname{reg}\left(I(H)^{q}\right)=2 q+\nu(H)+(t-1)-2$ for all $q \geq 2$. Note that $G_{1}=H \coprod C_{n_{t}}$. By [22, Proposition 2.7], $\operatorname{reg}\left(I\left(G_{1}\right)^{2}\right)=2+\nu\left(G_{1}\right)+t$. Using [8, Theorem 5.2] and [35, Theorem 1.1], we get $\operatorname{reg}\left(I\left(G_{1}\right)^{3}\right)=4+\nu\left(G_{1}\right)+t$. When $q \geq 4$, we can apply [35, Theorem 5.7] by taking $I=I(H)$ and $J=I\left(C_{n_{t}}\right)$. Note that $g=\nu(H)+t-3$, $g^{*}=\nu(H)+t-2, h=\nu\left(C_{n_{t}}\right)-1$ and $h^{*}=\nu\left(C_{n_{t}}\right)$ with notation of [35, Theorem 5.7]. Then we get $\operatorname{reg}\left(I\left(G_{1}\right)^{q}\right)=2 q+\nu\left(G_{1}\right)+t-2$ for all $q \geq 4$.

(2) It follows from [22, Proposition 2.7] that $\operatorname{reg}\left(I(G)^{2}\right)=3+\nu(G)+t$. Similarly to the previous case, by applying [35, Theorem 5.7] with $I=I\left(G_{1}\right)$ and $J=I\left(G_{2}\right)$, we have $\operatorname{reg}\left(I(G)^{q}\right)=2 q+\nu(G)+t-1$ for all $q \geq 3$.

Trung proved that for a graph $G, \operatorname{reg}(I(G))=\mathrm{m}(G)+1$ if and only if each connected component of $G$ is either $C_{5}$ or a Cameron-Walker graph, [41, Theorem 11]. As an immediate consequence of our previous result, we compute the regularity of all powers of such graphs.

Proposition 4.7. If $\operatorname{reg}(I(G))=\mathrm{m}(G)+1$, then either $\operatorname{reg}\left(I(G)^{q}\right)=2 q+\mathrm{m}(G)-2$ for all $q \geq 2$ or $\operatorname{reg}\left(I(G)^{q}\right)=2 q+\mathrm{m}(G)-1$ for all $q \geq 2$.

Proof. Since $\operatorname{reg}(I(G))=\mathrm{m}(G)+1$, by [41, Theorem 11], it follows that

$$
G=\left(\coprod_{i=1}^{t} C_{5}\right) \coprod\left(\coprod_{j=1}^{l} H_{j}\right),
$$

where $H_{j}$ are Cameron-Walker graphs, for some $t, l \geq 0$. Note that $\nu\left(\coprod_{i=1}^{t} C_{5}\right)=t=$ $\mathrm{m}\left(\coprod_{i=1}^{t} C_{5}\right)-t$ and $\nu\left(\coprod_{j=i}^{l} H_{j}\right)=\mathrm{m}\left(\coprod_{j=i}^{l} H_{j}\right)$. If $l=0$, then by Proposition 4.6 (1), $\operatorname{reg}\left(I(G)^{q}\right)=2 q+\nu(G)+t-2$ for all $q \geq 2$. Therefore $\operatorname{reg}\left(I(G)^{q}\right)=2 q+\mathrm{m}(G)-2$ for all $q \geq 2$. Suppose $t=0$. Then by Theorem 4.4 and [8, Theorem 4.5], $\operatorname{reg}\left(I(G)^{q}\right)=$ $2 q+\nu(G)-1=2 q+\mathrm{m}(G)-1$ for all $q \geq 2$. If $t>0$ and $l>0$, then by Proposition 4.6(2), for all $q \geq 2, \operatorname{reg}\left(I(G)^{q}\right)=2 q+\nu(G)+t-1=2 q+\nu\left(\coprod_{i=1}^{t} C_{5}\right)+\nu\left(\coprod_{j=1}^{l} H_{j}\right)+t-1=$ $2 q+\mathrm{m}(G)-1$. 
Using Proposition 4.6, we obtain a class of graphs for which the upper bound in Theorem 4.4(1) is attained.

Proposition 4.8. For $p \geq 0$ and $r>p$, let $H=\left(\coprod_{i=1}^{p} C_{n_{i}}\right) \amalg\left(\coprod_{j=p+1}^{r} C_{n_{j}}\right)$, where $n_{1}, \ldots, n_{p} \equiv 2(\bmod 3)$ and $n_{p+1}, \ldots, n_{r} \equiv 0,1(\bmod 3)$. Then for all $q \geq 1$,

$$
\operatorname{reg}\left(I(H)^{q}\right)=2 q+\zeta(H)-1 .
$$

Proof. If $q=1$, then by [45, Lemma 8] and [28, Theorem 7.6.28], we get $\operatorname{reg}(I(H))=$ $\nu(H)+p+1=\zeta(H)+1$. Suppose $p=0$. Using [8, Theorem 5.2] and [35, Theorem 5.7], we get $\operatorname{reg}\left(I(H)^{q}\right)=2 q+\nu(H)-1=2 q+\zeta(H)-1$ for all $q \geq 1$. When $p \neq 0$, we can apply Proposition 4.6(2) with $G_{1}=\coprod_{i=1}^{p} C_{n_{i}}$ and $G_{2}=\coprod_{j=p+1}^{r} C_{n_{j}}$ and get $\operatorname{reg}\left(I(H)^{q}\right)=2 q+\nu(H)+p-1=2 q+\zeta(H)-1$ for all $q \geq 2$.

In [29], the authors asked if there exists a graph $G$ with $2 q+\nu(G)-1<\operatorname{reg}\left(I(G)^{q}\right)<$ $2 q+\operatorname{co-chord}(G)-1$ for all $q \gg 0$, [29, Question 5.8]. We show that some of the graphs considered in Proposition 4.8 satisfy this inequality. Let $H$ be a graph as in Proposition 4.8, with $n_{j} \equiv 1(\bmod 3)$ and $n_{j}>4$ for $j=p+1, \ldots, r, p>0$. Then $\nu(H)=\sum_{i=1}^{r}\left\lfloor\frac{n_{i}}{3}\right\rfloor$, $\zeta(H)=p+\sum_{i=1}^{r}\left\lfloor\frac{n_{i}}{3}\right\rfloor$ and co-chord $(H)=r+\sum_{i=1}^{r}\left\lfloor\frac{n_{i}}{3}\right\rfloor$. Therefore, we get for all $q \geq 1$,

$$
2 q+\nu(H)-1<\operatorname{reg}\left(I(H)^{q}\right)=2 q+\zeta(H)-1<2 q+\operatorname{co}-\operatorname{chord}(H)-1 .
$$

\section{Regularity of POWERS OF VERTEX DECOMPOSABLE GRAPHS}

In this section, we prove Conjecture 1.2 for vertex decomposable graphs. We first recall the definition of a simplicial complex and a vertex decomposable graph.

A simplicial complex $\Delta$ on $V=\left\{x_{1}, \ldots, x_{n}\right\}$ is a collection of subsets of $V$ such that:

(1) $\left\{x_{i}\right\} \in \Delta$ for $i=1, \ldots, n$, and

(2) if $F \in \Delta$ and $G \subseteq F$, then $G \in \Delta$.

Elements of $\Delta$ are called the faces of $\Delta$, and the maximal elements, with respect to inclusion, are called the facets. The link of a face $F$ in $\Delta$ is $\operatorname{link}_{\Delta}(F)=\left\{F^{\prime} \in \Delta \mid\right.$ $\left.F^{\prime} \cup F \in \Delta, F^{\prime} \cap F=\emptyset\right\}$.

A simplicial complex $\Delta$ is recursively defined to be vertex decomposable if it is either a simplex or else has some vertex $v$ such that

(1) both $\Delta \backslash v$ and $\operatorname{link}_{\Delta} v$ are vertex decomposable, and

(2) no face of $\operatorname{link}_{\Delta} v$ is a facet of $\Delta \backslash v$.

The independence complex of $G$, denoted by $\Delta(G)$, is the simplicial complex on $V(G)$ with face set

$$
\Delta(G)=\{F \subseteq V(G) \mid F \text { is an independent set of } G\} .
$$

A graph $G$ is said to be vertex decomposable if $\Delta(G)$ is a vertex decomposable simplicial complex. In [43], Woodroofe translated the notion of vertex decomposable for graphs as follows.

Definition 5.1. [43, Lemma 4] A graph $G$ is recursively defined to be vertex decomposable if $G$ is totally disconnected (with no edges) or if there is a vertex $x$ in $G$ such that 
(1) $G \backslash x$ and $G \backslash N_{G}[x]$ are both vertex decomposable, and

(2) no independent set in $G \backslash N_{G}[x]$ is a maximal independent set in $G \backslash x$.

A vertex $x$ which satisfies the second condition of Definition 5.1 is called a shedding vertex of $G$. If $G$ is a vertex decomposable graph, then by [9, Theorem 2.5], $G \backslash N_{G}[x]$ is a vertex decomposable graph, for any $x \in V(G)$. For any vertex decomposable graph $K$, set

$\mathcal{S}(K)=\{x \in V(K) \mid x$ is a shedding vertex and $K \backslash x$ is a vertex decomposable graph $\}$.

Note that if $K$ is vertex decomposable, then $\mathcal{S}(K) \neq \emptyset$. The following observation is crucial for the proof of Theorem 5.3 .

Observation 5.2. Let $G$ be a vertex decomposable graph and $x \in \mathcal{S}(G)$. By [23, Theorem $4.2]$

$$
\operatorname{reg}(I(G))=\max \left\{\operatorname{reg}(I(G \backslash x)), \operatorname{reg}\left(I\left(G \backslash N_{G}[x]\right)\right)+1\right\} .
$$

Therefore, $\operatorname{reg}\left(I\left(G \backslash N_{G}[x]\right)\right)+1 \leq \operatorname{reg}(I(G))$.

We prove Conjecture 1.2 for the class of vertex decomposable graphs. Since induced subgraphs of a vertex decomposable graph are not necessarily be vertex decomposable, we cannot apply Theorem 4.1 to get the desired inequality. However, we can prove Conjecture 1.2 for the class of vertex decomposable graphs almost verbatim of the proof of Theorem 4.1 and we sketch the proof with the same notation as in the proof of Theorem 4.1.

Theorem 5.3. If $G$ is a vertex decomposable graph, then for all $q \geq 1$,

$$
\operatorname{reg}\left(I(G)^{q}\right) \leq 2 q+\operatorname{reg}(I(G))-2 .
$$

Proof. By applying [2, Theorem 5.2] and using induction on $q$, it is enough to prove that for any $s \geq 0$ and any minimal generator $M$ of $I(G)^{s}, \operatorname{reg}\left(I(G)^{s+1}: M\right) \leq \operatorname{reg}(I(G))$. Let $G^{\prime}$ be the graph associated to the ideal $\left(I(G)^{s+1}: e_{1} \cdots e_{s}\right)$ which is contained in an appropriate polynomial ring $R_{1}$, where $e_{1}, \ldots, e_{s} \in E(G)$. We prove that $\operatorname{reg}\left(I\left(G^{\prime}\right)\right) \leq$ $\operatorname{reg}(I(G))$ by induction on $s+|V(G)|$ which completes the proof. When either $s=0$ or $|V(G)|=2$, we have $G^{\prime}=G$ and the assertion is clear. Now, assume that $s \geq 1$ and $|V(G)|>2$. Let $e_{i}=\left\{a_{i}, b_{i}\right\}$. If $\operatorname{deg}_{G}\left(a_{i}\right)=1$ or $\operatorname{deg}_{G}\left(b_{i}\right)=1$ for some $i$, then the assertion follows as in the proof of Theorem 4.1. Therefore, we may assume that $\operatorname{deg}_{G}\left(a_{i}\right) \geq 2$ and $\operatorname{deg}_{G}\left(b_{i}\right) \geq 2$ for all $1 \leq i \leq s$.

CASE 1: Suppose $e_{i} \cap \mathcal{S}(G) \neq \emptyset$, for some $1 \leq i \leq s$.

Without loss of generality, assume that $e_{s} \cap \mathcal{S}(G) \neq \emptyset$ and $a_{s} \in \mathcal{S}(G)$. Now proceeding as in the proof Theorem 4.1 with the same notation, one gets

$$
\begin{aligned}
\operatorname{reg}\left(J+\left(\mathcal{X}_{b_{s}}\right)\right) & \leq \operatorname{reg}\left(I\left(\left(G \backslash N_{G}\left[b_{s}\right]\right)^{\prime}\right)\right) \leq \operatorname{reg}\left(I\left(G \backslash N_{G}\left[b_{s}\right]\right)\right) \leq \operatorname{reg}(I(G)) \\
\operatorname{reg}\left(J: y_{i}\right) & \leq \operatorname{reg}\left(I\left(\left(G \backslash N_{G}\left[y_{i}, a_{s}\right]\right)^{\prime}\right)\right) \leq \operatorname{reg}\left(I\left(\left(G \backslash N_{G}\left[a_{s}\right]\right)^{\prime}\right)\right) \\
& \leq \operatorname{reg}\left(I\left(G \backslash N_{G}\left[a_{s}\right]\right)\right)<\operatorname{reg}(I(G)) .
\end{aligned}
$$

Here, we use Lemmas 3.6, 3.7 and inductive hypothesis (since $G \backslash N_{G}\left[b_{s}\right]$ and $G \backslash N_{G}\left[a_{s}\right]$ are vertex decomposable graphs) along with Observation 5.2 for the above conclusions.

Using these inequalities, we conclude, as in the proof of Theorem 4.1, that $\operatorname{reg}(J) \leq$ $\operatorname{reg}(I(G))$. 
CASE 2: Suppose $e_{i} \cap \mathcal{S}(G)=\emptyset$, for all $1 \leq i \leq s$. Let $x \in \mathcal{S}(G)$. By [21, Theorem 3.4], $\operatorname{reg}\left(I\left(G^{\prime}\right)\right) \leq \max \left\{\operatorname{reg}\left(I\left(G^{\prime} \backslash x\right)\right), \operatorname{reg}\left(I\left(G^{\prime} \backslash N_{G^{\prime}}[x]\right)+1\right\}\right.$. Since $G \backslash x$ is vertex decomposable, we can use Lemmas 3.3 , 3.5 along with Observation 5.2 to derive $\operatorname{reg}\left(I\left(G^{\prime}\right)\right) \leq \operatorname{reg}(I(G))$ as done in the proof of CASE 2 in Theorem 4.1 .

This completes the proof.

As an immediate consequence of the above result, we obtain the linear polynomial corresponding to $\operatorname{reg}\left(I(G)^{q}\right)$ for several classes of graphs. A simplicial vertex of a graph $G$ is a vertex $x$ such that the neighbors of $x$ form a complete subgraph in $G$.

Corollary 5.4. Let $G$ be a graph with one of the following properties:

(1) vertex decomposable and contains no 5-cycles;

(2) vertex decomposable and contains no induced 5-cycles and 4-cycles;

(3) for any independent set $A$, the graph $G \backslash N_{G}[A]$ is a collection of isolated vertices or has a simplicial vertex of degree at least one;

(4) sequentially Cohen-Macaulay bipartite;

(5) obtained from a graph $H$ by adding whiskers on a subset $S$ of the vertex set of $H$ such that $H \backslash S$ is chordal.

Then for all $q \geq 1$,

$$
\operatorname{reg}\left(I(G)^{q}\right)=2 q+\nu(G)-1 .
$$

Proof. (1) \& (2): From [32, Theorem 2.4] and [10, Theorem 24], it follows that for these classes of graph, $\operatorname{reg}(I(G))=\nu(G)+1$. Hence the assertion follows from [8, Theorem 4.5] and Theorem 5.3 .

(3) First we prove that $\operatorname{reg}(I(G)) \leq \nu(G)+1$, by induction on $|V(G)|$. Since, by our assumption, $G$ does not have isolated vertices, the assertion is immediate for the base case $|V(G)|=2$. Assume now that $|V(G)|>2$. Since the empty set is independent, there is a simplicial vertex $x$ in $G$. Let $N_{G}(x)=\left\{x_{1}, \ldots, x_{m}\right\}$ with $m \geq 1$. Set $I(G)=I_{0}$ and $I_{l}=I_{0}+\left(x_{1}, \ldots, x_{l}\right)$ for $1 \leq l \leq m$. Then, for $0 \leq l \leq m-1$, we have

$$
0 \longrightarrow \frac{R}{\left(I_{l}: x_{l+1}\right)}(-1) \stackrel{x_{l+1}}{\longrightarrow} \frac{R}{I_{l}} \longrightarrow \frac{R}{I_{l}+\left(x_{l+1}\right)} \longrightarrow 0 .
$$

Therefore,

$$
\operatorname{reg}\left(I_{0}\right) \leq \max \left\{\operatorname{reg}\left(I_{m}\right), \operatorname{reg}\left(I_{l}: x_{l+1}\right)+1: 0 \leq l \leq m-1\right\} .
$$

Since $\left\{x_{1}, \ldots, x_{l}\right\} \subset N_{G}\left[x_{l+1}\right],\left(\left(G \backslash N_{G}\left[x_{l+1}\right]\right) \backslash\left\{x_{1}, \ldots, x_{l}\right\}\right)=G \backslash N_{G}\left[x_{l+1}\right]$ for any $0 \leq$ $l \leq m-1$. Hence $\left(I_{l}: x_{l+1}\right)=\left(I_{0}: x_{l+1}\right)$. It is easy to see that for any vertex $u$ of $G$ and an independent set $B$ of $G \backslash N_{G}[u]$, the set $B \cup\{u\}$ is an independent set of $G$. Then $G \backslash N_{G}[u]$ is also a graph with the property (3). Therefore, we may apply inductive hypothesis to get

$$
\operatorname{reg}\left(I_{m}\right)=\operatorname{reg}\left(I\left(G \backslash N_{G}[x]\right)\right) \leq \nu\left(G \backslash N_{G}[x]\right)+1 \leq \nu(G)+1
$$

and for any $0 \leq l \leq m-1$,

$$
\operatorname{reg}\left(I_{l}: x_{l+1}\right)=\operatorname{reg}\left(I_{0}: x_{l+1}\right)=\operatorname{reg}\left(I\left(G \backslash N_{G}\left[x_{l+1}\right]\right)\right) \leq \nu\left(G \backslash N_{G}\left[x_{l+1}\right]\right)+1 .
$$

If $\left\{f_{1}, \ldots, f_{t}\right\}$ is an induced matching of $G \backslash N_{G}\left[x_{l+1}\right]$, then $\left\{f_{1}, \ldots, f_{t},\left\{x, x_{l+1}\right\}\right\}$ is an induced matching of $G$. Therefore $\nu\left(G \backslash N_{G}\left[x_{l+1}\right]\right)+1 \leq \nu(G)$. Hence reg $(I(G)) \leq \nu(G)+1$. 
By [44, Corollary 5.5], $G$ is a vertex decomposable graph. Therefore, by Theorem 5.3 and [8, Theorem 4.5], $\operatorname{reg}\left(I(G)^{q}\right)=2 q+\nu(G)-1$.

(4) By [42, Theorem 2.10], $G$ is vertex decomposable. Since a bipartite graph contains no 5 -cycles, the assertion follows from (1).

(5) In [18], Dirac proved that a graph $L$ is chordal if and only if every induced subgraph of $L$ has a simplicial vertex. Let $A$ be any independent set of $G$. If $S \subseteq N_{G}[A]$, then $G \backslash N_{G}[A]$ is chordal or isolated vertices. If $S \varsubsetneqq N_{G}[A]$, then $G \backslash N_{G}[A]$ has atleast one whisker and hence $G \backslash N_{G}[A]$ has a simplicial vertex. Therefore $G \backslash N_{G}[A]$ has a simplicial vertex for any independent set $A$. Hence, by $(3), \operatorname{reg}\left(I(G)^{q}\right)=2 q+\nu(G)-1$ for all $q \geq 1$.

Acknowledgement: We would like to thank Fahimeh Khosh-Ahang, Huy Tài Hà, Adam Van Tuyl and Russ Woodroofe for several clarifications on our doubts on their results. We also would like to thank Arindam Banerjee and Huy Tài Hà for pointing out an error in one of the proofs in an earlier version of the manuscript. We extensively used MACAULAY2, [20], SAGE, [17] and the package SimplicialDecomposability, [15], for testing our computations. The second author is partially supported by DST, Govt of India under the DST-INSPIRE Faculty Scheme. We would also like to express our sincere gratitude to anonymous referees for meticulous reading and suggesting several improvements.

\section{REFERENCES}

[1] A. Alilooee and A. Banerjee. Powers of edge ideals of regularity three bipartite graphs. J. Commut. Algebra, 9(4):441-454, 2017.

[2] A. Banerjee. The regularity of powers of edge ideals. J. Algebraic Combin., 41(2):303-321, 2015.

[3] A. Banerjee, S. K. Beyarslan, and H. T. Hà. Regularity of edge ideals and their powers. In Advances in algebra, volume 277 of Springer Proc. Math. Stat., pages 17-52. Springer, Cham, 2019.

[4] A. Banerjee, S. K. Beyarslan, and H. T. Hà. Regularity of powers of edge ideals: from local properties to global bounds. Algebr. Comb., 3(4):839-854, 2020.

[5] A. Banerjee and E. Nevo. Regularity of Edge Ideals Via Suspension. arXiv e-prints, arXiv:1908.03115, Aug 2019.

[6] D. Berlekamp. Regularity defect stabilization of powers of an ideal. Math. Res. Lett., 19(1):109-119, 2012.

[7] A. Bertram, L. Ein, and R. Lazarsfeld. Vanishing theorems, a theorem of Severi, and the equations defining projective varieties. J. Amer. Math. Soc., 4(3):587-602, 1991.

[8] S. Beyarslan, H. T. Hà, and T. N. Trung. Regularity of powers of forests and cycles. J. Algebraic Combin., 42(4):1077-1095, 2015.

[9] J. Biermann, C. A. Francisco, H. T. Hà, and A. Van Tuyl. Partial coloring, vertex decomposability, and sequentially Cohen-Macaulay simplicial complexes. J. Commut. Algebra, 7(3):337-352, 2015.

[10] T. Biyıkoğlu and Y. Civan. Vertex-decomposable graphs, codismantlability, Cohen-Macaulayness, and Castelnuovo-Mumford regularity. Electron. J. Combin., 21(1):Paper 1.1, 17pp, 2014.

[11] A. Björner and M. L. Wachs. Shellable nonpure complexes and posets. II. Trans. Amer. Math. Soc., 349(10):3945-3975, 1997.

[12] A. H. Busch, F. F. Dragan, and R. Sritharan. New min-max theorems for weakly chordal and dually chordal graphs. In Combinatorial optimization and applications. Part II, volume 6509 of Lecture Notes in Comput. Sci., pages 207-218. Springer, Berlin, 2010.

[13] K. Cameron and T. Walker. The graphs with maximum induced matching and maximum matching the same size. Discrete Math., 299(1-3):49-55, 2005.

[14] K. A. Chandler. Regularity of the powers of an ideal. Comm. Algebra, 25(12):3773-3776, 1997.

[15] D. Cook, II. Simplicial decomposability. J. Softw. Algebra Geom., 2:20-23, 2010. 
[16] S. D. Cutkosky, J. Herzog, and N. V. Trung. Asymptotic behaviour of the Castelnuovo-Mumford regularity. Compositio Math., 118(3):243-261, 1999.

[17] T. S. Developers. Sage Mathematics Software (Version 6.9), 2015. http://www. sagemath.org.

[18] G. A. Dirac. On rigid circuit graphs. Abh. Math. Sem. Univ. Hamburg, 25:71-76, 1961.

[19] A. V. Geramita, A. Gimigliano, and Y. Pitteloud. Graded Betti numbers of some embedded rational $n$-folds. Math. Ann., 301(2):363-380, 1995.

[20] D. R. Grayson and M. E. Stillman. Macaulay2, a software system for research in algebraic geometry. Available at http://www.math.uiuc.edu/Macaulay2/.

[21] H. T. Hà. Regularity of squarefree monomial ideals. In Connections between algebra, combinatorics, and geometry, volume 76 of Springer Proc. Math. Stat., pages 251-276. Springer, New York, 2014.

[22] H. T. Hà, N. V. Trung, and T. N. Trung. Depth and regularity of powers of sums of ideals. Math. Z., 282(3-4):819-838, 2016.

[23] H. T. Hà and R. Woodroofe. Results on the regularity of square-free monomial ideals. Adv. in Appl. Math., 58:21-36, 2014.

[24] J. Herzog and T. Hibi. Monomial ideals, volume 260 of Graduate Texts in Mathematics. SpringerVerlag London, Ltd., London, 2011.

[25] J. Herzog, T. Hibi, and X. Zheng. Monomial ideals whose powers have a linear resolution. Math. Scand., 95(1):23-32, 2004.

[26] T. Hibi, A. Higashitani, K. Kimura, and A. B. O'Keefe. Algebraic study on Cameron-Walker graphs. J. Algebra, 422:257-269, 2015.

[27] T. Hibi, A. Higashitani, K. Kimura, and A. Tsuchiya. Dominating induced matchings of finite graphs and regularity of edge ideals. J. Algebraic Combin., 43(1):173-198, 2016.

[28] S. Jacques. Betti numbers of graph ideals. PhD thesis, University of Sheffield, 2004.

[29] A. V. Jayanthan, N. Narayanan, and S. Selvaraja. Regularity of powers of bipartite graphs. J. Algebraic Combin., 47(1):17-38, 2018.

[30] A. V. Jayanthan and S. Selvaraja. Linear polynomials for the regularity of powers of edge ideals of very well-covered graphs. J. Commut. Algebra (to appear), arXiv e-prints, arXiv:1708.06883, Aug 2017.

[31] M. Katzman. Characteristic-independence of Betti numbers of graph ideals. J. Combin. Theory Ser. A, 113(3):435-454, 2006.

[32] F. Khosh-Ahang and S. Moradi. Regularity and projective dimension of the edge ideal of $C_{5}$-free vertex decomposable graphs. Proc. Amer. Math. Soc., 142(5):1567-1576, 2014.

[33] V. Kodiyalam. Asymptotic behaviour of Castelnuovo-Mumford regularity. Proc. Amer. Math. Soc., 128(2):407-411, 2000.

[34] S. Morey. Depths of powers of the edge ideal of a tree. Comm. Algebra, 38(11):4042-4055, 2010.

[35] H. D. Nguyen and T. Vu. Powers of sums and their homological invariants. J. Pure Appl. Algebra, 223(7):3081-3111, 2019.

[36] J. S. Provan and L. J. Billera. Decompositions of simplicial complexes related to diameters of convex polyhedra. Math. Oper. Res., 5(4):576-594, 1980.

[37] T. Römer. Homological properties of bigraded algebras. Illinois J. Math., 45(4):1361-1376, 2001.

[38] S. A. Seyed Fakhari and S. Yassemi. Improved bounds for the regularity of powers of edge ideals of graphs. arXiv e-prints, arXiv:1805.12508, May 2018.

[39] I. Swanson. Powers of ideals. Primary decompositions, Artin-Rees lemma and regularity. Math. Ann., 307(2):299-313, 1997.

[40] N. V. Trung and H.-J. Wang. On the asymptotic linearity of Castelnuovo-Mumford regularity. $J$. Pure Appl. Algebra, 201(1-3):42-48, 2005.

[41] T. N. Trung. Regularity, matchings and Cameron-Walker graphs. Collect. Math., 71(1):83-91, 2020.

[42] A. Van Tuyl. Sequentially Cohen-Macaulay bipartite graphs: vertex decomposability and regularity. Arch. Math. (Basel), 93(5):451-459, 2009.

[43] R. Woodroofe. Vertex decomposable graphs and obstructions to shellability. Proc. Amer. Math. Soc., 137(10):3235-3246, 2009.

[44] R. Woodroofe. Chordal and sequentially Cohen-Macaulay clutters. Electron. J. Combin., 18(1):Paper 208, 20pp, 2011. 
[45] R. Woodroofe. Matchings, coverings, and Castelnuovo-Mumford regularity. J. Commut. Algebra, 6(2):287-304, 2014.

Email address: jayanav@iitm.ac.in

Department of Mathematics, Indian Institute of Technology Madras, Chennai, India $-600036$

Email address: selva.y2s@gmail.com

Chennai Mathematical Institute, H1, SipCOT it Park, Siruseri, Kelambakkam, ChenNAI, INDIA - 603103. 